\title{
Analysis and Simulation of SIR Epidemic Model by Considering Comorbidities
}

\author{
Yudi Ari Adi ${ }^{1}$ Dita Nur Avina ${ }^{2}$ Ario Wiraya ${ }^{3, *}$ Laila Fitriana $^{4}$ Triyanto $^{5}$ \\ 1,2 Department of Mathematics, Ahmad Dahlan University, Yogyakarta, Indonesia \\ 3,4,5 Department of Mathematics Education, Sebelas Maret University, Surakarta, Indonesia \\ *Corresponding author.Email: ariowiraya@staff.uns.ac.id
}

\begin{abstract}
Comorbidities have an influence on progression of infectious disease suffered by the patients. In this paper, we construct and analyze the SIR epidemic model by considering comorbidities in infected patients to characterize the infectious disease progression with comorbidities intervention as a consideration in determining the appropriate treatment. By determining the formula of the basic reproduction number, the conditions which represent stability of the disease-free equilibrium point and the disease equilibrium point are identified. Furthermore, the sufficient and necessary condition for local and global stability of disease-free equilibrium points and local stability of both non-comorbidities and comorbidities endemic equilibrium points are investigated. Theoretical findings of this research are supported by performing numerical simulations as an illustration of the dynamics which occur on the model solution.
\end{abstract}

Keywords: Epidemic model, SIR, Comorbidities, Simulation.

\section{INTRODUCTION}

In epidemiology, mathematical models that explain the dynamics of human infectious diseases have recently played a key role in disease control, particularly the currently ongoing disease, namely COVID-19. The pandemic of the new coronavirus named coronavirus 2019 (COVID-19) is currently still ongoing and still cannot be completely stopped. This virus has been transmitted rapidly throughout the world, including in Indonesia. The COVID-19 had $190,289,510$ confirmed cases worldwide as of July 19, 2021, with 4,092,361 deaths (CFR 2.2\%) in 204 infected nations and 151 community transmission countries. Meanwhile, the Government of the Republic of Indonesia has recorded 2,911,733 confirmed cases of COVID-19, 74,920 deaths (CFR: 2.6 percent) from the disease, and 2,293,875 patients who have recovered [1].

Understanding the process of virus transmission is essential to determine the best virus mitigation techniques. Reducing the transmission rate [2], such as the use of masks, social distancing, and handwashing, as well as detecting infected individuals, are strategies to reduce the number of infection $[3,4,5]$. The effort of early detection in reducing the burden of disease must always be carried out. However, it must be acknowledged that lack of resources can make it difficult to reduce the incidence of infection [6].

Various mathematical models have been developed to get a better understanding of the dynamics of COVID-19 disease transmission and the efficacy of therapeutic strategies $[7,8,9,10]$. Many models have been developed to assess the effectiveness of intervention $[6,8,10,11]$ and investigate the patterns of infectious disease progression [12]. Despite the fact that several mathematical models have been developed to explore the dynamics of COVID-19 transmission, research on COVID-19 transmission which has focused on individuals with comorbidities is still limited, even though patients with comorbidities, such as hypertension or diabetes mellitus, are have a higher risk of undergoing severe symptoms and death [13].

Many epidemic models have been devised by researchers to get a better understanding on the mechanism of disease transmission. Since the famous Kermack-McKendrick SIR model had been introduced, various epidemic models have been defined and explored in various types of literature. However, the SIR model that differentiates the 
infection population is still very limited. In this paper, we will formulate and analyze a mathematical model for disease transmission based on SIR epidemic model by distinguishing patients with and without comorbidities. Analysis of the model can be implemented to determine the progression of infectious diseases that differentiate between classes of infection, such as COVID-19 as well as the other diseases.

\section{MODEL FORMULATION}

\subsection{The Model}

On the formation of a mathematical model of COVID-19 transmission, the population is divided into four subpopulations, namely Susceptible $(S)$, infected without comorbidities ( $I$ ), infected with comorbidities $\left(I_{p}\right)$, and Recovered $(R)$. The transmission scheme is presented in the compartment diagram in Figure 1.

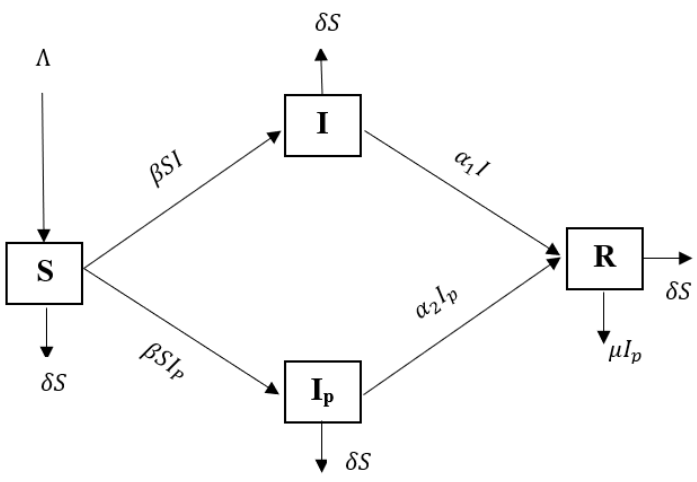

Figure 1 Schematic diagram for the COVID-19 disease.

It is assumed that the susceptible individuals are recruited into the population at a constant rate $\Lambda$. Susceptible individuals are reduced by infection, following primary contact with infected individuals with or without comorbidities at the rate $\beta$, and a natural death rate $\delta$. Therefore, the rate of change of the susceptible individuals is given by

$$
\frac{d S}{d t}=\Lambda-\beta S\left(I+I_{P}\right)-\delta S .
$$

The population of infected individuals without comorbidities is increased by infection of susceptible individuals at a rate of $\beta$. Infected individuals without comorbidities disease will decrease with the curing rate $\alpha_{1}$ and natural death rate $\delta$ Therefore, the following equation is obtained.

$$
\frac{d I}{d t}=\beta S I-\alpha_{1} I-\delta I \text {. }
$$

The population of individuals infected with comorbidities is increased by infection of susceptible individuals at a rate of $\beta$. Infected individuals with comorbidities will decrease with the cure rate $\alpha_{1}$, death due to covid infection at a rate of $\alpha_{2}$, and the natural death rate $\delta$. Therefore, mathematically, it is

$$
\frac{d I_{P}}{d t}=\beta S I_{P}-\alpha_{2} I_{P}-\mu I_{p}-\delta I_{p} .
$$

Furthermore, the recovered population comes from individuals who recover from infection with or without comorbidities and decrease due to natural deaths. The corresponding differential equation is given by

$$
\frac{d R}{d t}=\alpha_{1} I+\alpha_{2} I_{P}-\delta R
$$

Thus, the model of COVID-19 transmission with comorbidities is given by the following nonlinear differential system:

$$
\begin{aligned}
& \frac{d S}{d t}=\Lambda-\beta S\left(I+I_{P}\right)-\delta S \\
& \frac{d I}{d t}=\beta S I-\alpha_{1} I-\delta I \\
& \frac{d I_{P}}{d t}=\beta S I_{P}-\alpha_{2} I_{P}-\mu I_{p}-\delta I_{p} \\
& \frac{d R}{d t}=\alpha_{1} I+\alpha_{2} I_{P}-\delta R .
\end{aligned}
$$

The initial conditions of system (1) are $S(0), I(0), I_{p}(0)$, and $R(0)$.

\subsection{Properties of solutions}

Because (1) is a model that tracks the changes of human population, all parameters are assumed to be positive for all $t>0$. We also established that the solution of system (1) is non-negative by stating and proving the following theorem.

Theorem 2.1. Let $S(0), I(0), I_{p}(0)$, and $R(0)$ are positive, then the solution of the system (1) is nonnegative for all $t>0$.

Proof. From the first equation of the system (1) we have

$$
\begin{gathered}
\frac{d}{d t}\left(S(t) \exp \left(\int_{0}^{t} \beta\left(I(\tau)+I_{P}(\tau)\right) d \tau+\delta t\right)\right) \\
\quad=\Lambda \exp \left(\int_{0}^{t} \beta\left(I(\tau)+I_{P}(\tau)\right) d \tau+\delta t\right) .
\end{gathered}
$$


Integrating both sides from $t=0$ to $t=T$, we obtain

$$
\begin{gathered}
S(T) \exp \left(\int_{0}^{t} \beta\left(I(\tau)+I_{P}(\tau)\right) d \tau+\delta t\right)-S(0) \\
=\int_{0}^{T} \Lambda \exp \left(\int_{0}^{t} \beta\left(I(\tau)+I_{P}(\tau)\right) d \tau+\delta t\right) .
\end{gathered}
$$

Hence,

$$
\begin{aligned}
& S(T)=S(0) \exp \left(-\int_{0}^{t} \beta\left(I(\tau)+I_{P}(\tau)\right) d \tau+\delta t\right) \\
& \quad+\exp \left(-\int_{0}^{t} \beta\left(I(\tau)+I_{P}(\tau)\right) d \tau+\delta t\right) \\
& \times \int_{0}^{T} \Lambda \exp \left(\int_{0}^{t} \beta\left(I(\tau)+I_{P}(\tau)\right) d \tau+\delta t\right) d z>0,
\end{aligned}
$$

since the initial condition $S(0)>0$.

Similarly, it can be shown that $, I(0)>0, I_{p}(0)>0$, and $R(0)>0$ for all $t>0$.

Theorem 2.2. Let $S(t), I(t), I_{p}(t)$, and $R(t)$ be the solution of the system (1) with non-negative initial condition $S(0), I(0), I_{p}(0)$, and $R(0)$. The set of biological feasible region $\Omega=\left\{\left(S, I, I_{p}, R\right) \in\right.$ $\left.\mathbb{R}_{+}^{4} \mid N=\Lambda / \delta\right\}$ is positively invariant.

Proof. Since the total population $N(t)=S(t)+$ $I(t)+I_{p}(t)+R(t)$, we get

$$
\frac{d N}{d t}=\Lambda-\mu I_{p}-\delta N \leq \Lambda-\delta N .
$$

Thus, we have $0 \leq \lim _{t \rightarrow \infty} \sup N(t) \leq \Lambda / \delta$ so all solution of system (1) ultimately enters the closed set $\Omega$. Hence, the region $\Omega$ is positively invariant

\subsection{Existence and stability of equilibria}

The system (1) always has a disease-free equilibrium point which is found by setting the righthand side of the equation in (1) to zero, i. e.

$$
E_{0}=\left(\frac{\Lambda}{\delta}, 0,0,0\right) .
$$

The basic reproduction number of the system (1) is

$$
R_{0}=\max \left\{R_{1}, R_{2}\right\},
$$

with $\quad R_{1}=\frac{\beta \Lambda}{\delta\left(\alpha_{1}+\delta\right)} \quad$ and $\quad R_{2}=\frac{\beta \Lambda}{\delta\left(\alpha_{2}+\mu+\delta\right)} \quad$ which obtained by using the next generation matrix procedure described in [14].
The stability of the disease-free equilibrium point is stated in the following theorem.

Theorem 2.3. The disease-free equilibrium point $E_{0}$ is locally asymptotically stable if $R_{0}<1$ and unstable if $R_{0}>1$.

Proof. The Jacobian matrix of the system (1) at the disease-free equilibrium point $E_{0}=\left(\frac{\Lambda}{\delta}, 0,0,0\right)$ is

$J\left(E_{0}\right)=$

$\left[\begin{array}{cccc}-\delta & -\frac{\beta \Lambda}{\delta} & -\frac{\beta \Lambda}{\delta} & 0 \\ 0 & \frac{\beta \Lambda}{\delta}-\left(\alpha_{1}+\delta\right) & 0 & 0 \\ 0 & 0 & \frac{\beta \Lambda}{\delta}-\left(\alpha_{2}+\mu+\delta\right) & 0 \\ 0 & \alpha_{1} & \alpha_{2} & -\delta\end{array}\right]$

The eigenvalues of the Jacobian matrix $J\left(E_{0}\right)$ are $\lambda_{1}=-\delta, \lambda_{2}=\left(\alpha_{1}+\delta\right)\left(R_{1}-1\right), \lambda_{3}=\left(\alpha_{2}+\mu+\right.$ $\delta)\left(R_{2}-1\right)$, and $\lambda_{4}=-\delta$. Hence, all eigenvalues are negative if only in $R_{1}<1$ and $R_{2}<1$. From (3), it is concluded that, $E_{0}$ is locally asymptotically stable if $R_{0}<1$ and unstable if $R_{0}>1$

Furthermore, when $R_{0}<1$, we can verify that $E_{0}$ is global stability.

Theorem 2.4. The disease-free equilibrium point $E_{0}$ is globally asymptotically stable if $R_{0}<1$.

Proof. We define the Lyapunov function to investigate whether the endemic equilibrium is globally asymptotically stable as follows

$$
L=\delta I+\alpha_{1} I_{p}
$$

Clearly, $L$ is positive definite and we obtain the derivative

$$
\begin{aligned}
\frac{d L}{d t} & =\delta \frac{d I}{d t}+\alpha_{1} \frac{d I_{p}}{d t} \\
& =\delta\left(\beta S I-\left(\alpha_{1}+\delta\right) I\right)+\alpha_{1}\left(\beta S I_{P}-\left(\alpha_{2}+\mu+\delta\right) I_{p}\right) \\
& \leq \delta I\left(\frac{\beta \Lambda}{\delta}-\left(\alpha_{1}+\delta\right)\right)+\alpha_{2} I_{p}\left(\frac{\beta \Lambda}{\delta}-\left(\alpha_{2}+\mu+\delta\right)\right) \\
= & \delta I\left(\alpha_{1}+\delta\right)\left(R_{1}-1\right)+\alpha_{2} I_{p}\left(\alpha_{2}+\mu+\delta\right)\left(R_{2}-1\right) .
\end{aligned}
$$

Since $R_{0}<1$, it follows that $\frac{d L}{d t} \leq 0$ and $\frac{d L}{d t}=0$ if $I=I_{p}=0$. Hence, $L$ is a Lyapunov function and the largest compact invariant set is the singleton $\left\{E_{0}\right\}$. Thus, by LaSalle-Lyapunov Theorem [15], $\left\{E_{0}\right\}$ is globally asymptotically stable.

The system (1) has a non-comorbidities dominance equilibrium point $E_{1}$, in which infected individuals without comorbidities $I \neq 0$, while the infected individuals with comorbidities $I_{p}=0$. The non- 
comorbidities dominance equilibrium point is given by

$$
E_{1}=\left(\frac{\alpha_{1}+\delta}{\beta}, \frac{\delta}{\beta}\left(R_{1}-1\right), 0, \frac{\alpha_{1}}{\beta}\left(R_{1}-1\right)\right) .
$$

The system (1) also has comorbidities dominance equilibrium point $E_{2}$, in which infected individuals without comorbidities $I=0$, while the infected individuals with comorbidities $I_{p} \neq 0$ that given by

$$
E_{2}=\left(\frac{\alpha_{2}+\mu+\delta}{\beta}, 0, \frac{\delta}{\beta}\left(R_{2}-1\right), \frac{\alpha_{2}}{\beta}\left(R_{2}-1\right)\right) .
$$

From (4) and (5) we know that the noncomorbidities dominance equilibrium point $E_{1}$ exist if $R_{1}>1$ and the comorbidities dominance equilibrium point $E_{2}$ exist if $R_{2}>1$. Hence, if both $R_{1}$ and $R_{2}$ are greater than one. there coexistence of the noncomorbidities dominance equilibrium point $E_{1}$ and the comorbidities dominance equilibrium point $E_{2}$. For stability these equilibria, we have the following stability theorem.

Theorem 2.5. Let $R_{1}>1$, and $R_{2}>1$.

i The non-comorbidities dominance equilibrium point $E_{1}$ is locally asymptotically stable if $R_{2}<R_{1}$ and unstable if $R_{1}<R_{2}$.

ii If $R_{1}<R_{2}$ then the comorbidities dominance equilibrium point $E_{2}$ is locally asymptotically and unstable if $R_{2}<R_{1}$.

Proof. The eigenvalues of the Jacobian matrix of the system (1) at $E_{1}$ are $\lambda_{1}=-\delta$, which always negative and $\lambda_{2}=\left(\alpha_{2}+\mu\right)-\delta$. The remaining eigenvalues are the roots of a quadratic equation $\lambda^{2}+\delta R_{2} \lambda+$ $\left(\alpha_{2}+\mu+\delta\right) \delta\left(R_{2}-1\right)=0$, which have roots with negative real part if $R_{2}>1$. Thus, all eigenvalues have negative real parts if $\left(\alpha_{2}+\mu\right)-\delta$ which will be fulfilled if $R_{2}<R_{1}$. Hence, the non-comorbidities dominance equilibrium point $E_{1}$ is locally asymptotically stable if $\mathrm{R}_{2}<\mathrm{R}_{1}$ and unstable if $\mathrm{R}_{1}<$ $R_{2}$. The proof of the stability of dominance equilibrium point $\mathrm{E}_{2}$ is similar.

\section{SIMULATION RESULTS}

In this section, we investigate the numerical results to verify the mathematical analysis and to investigate the behaviour of the solution of system (1).

\subsection{The stability of disease-free equilibrium}

We set a parameter values

$$
\begin{gathered}
\Lambda=30, \beta=0.8 \times 10^{-7}, \alpha_{1}=0.83, \alpha_{2}=0.75, \\
\mu=0.03, \delta=3.64 \times 10^{-5} .
\end{gathered}
$$

With the set parameter values in (6), the condition of Theorem 2.3 is satisfied. We have $R_{1}=0.79435$ and $R_{2}=0.84526 \quad, \quad$ so that $R_{0}=$ $\max \{0.79435,0.84526\}=0.84526$. In this case, we obtain the disease-free equilibrium point $E_{0}=$ $(824,175,0,0,0)$ which is globally asymptotically stable, see Figure 2. Figure 2a shows the time series solution, while Figure $2 \mathrm{~b}$ shows that all trajectories with various initial conditions tend to the disease-free equilibrium point.
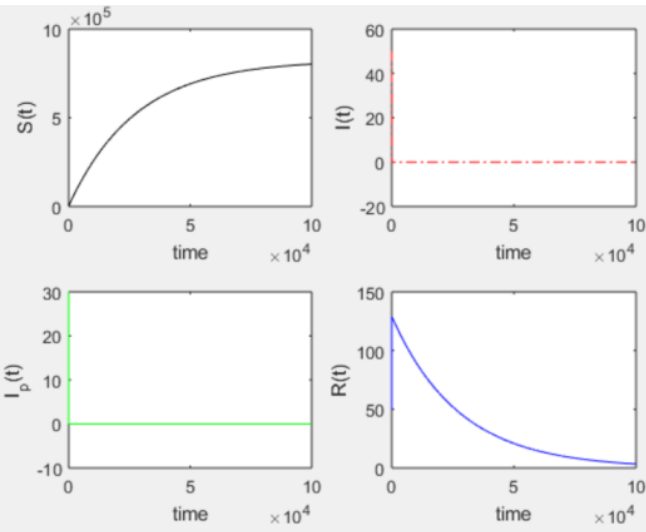

(a) Time series of $E_{0}$

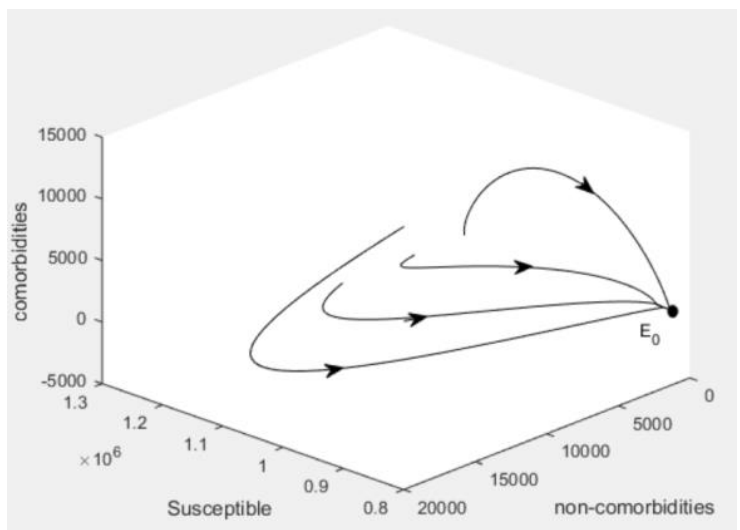

(b) Trajectories solution of $E_{0}$.

Figure 2 With the set of parameter values in (6), the disease-free equilibrium point $E_{0}$ is globally asymptotically stable.

\subsection{The stability of endemic equilibrium}

\subsubsection{The non-comorbidities dominance}

In this case, we set the parameter values

$$
\begin{gathered}
\Lambda=30, \beta=0.8 \times 10^{-7}, \alpha_{1}=0.3, \alpha_{2}=0.3, \\
\mu=0.1, \delta=3.64 \times 10^{-5} .
\end{gathered}
$$


With the set parameter values in (7), we have $R_{1}=$ $2.19753>R_{2}=1.6482$ so that $R_{0}=R_{1}=2.19753$ and the condition in Theorem 2.5(i) is satisfied. So, in this case, we have the unstable disease-free equilibrium point $E_{0}=(824,175,0,0,0)$, the stable non-comorbidities endemic equilibrium $E_{1}=$ $(375,045.5,54.48,0,449,075.8)$, and the unstable comorbidities endemic equilibrium $E_{2}=$ $(500,045.5,0,29.49,243,075.6)$. Figure 3 shows the time series and trajectories solution of the stable noncomorbidities endemic equilibrium point $E_{1}$.
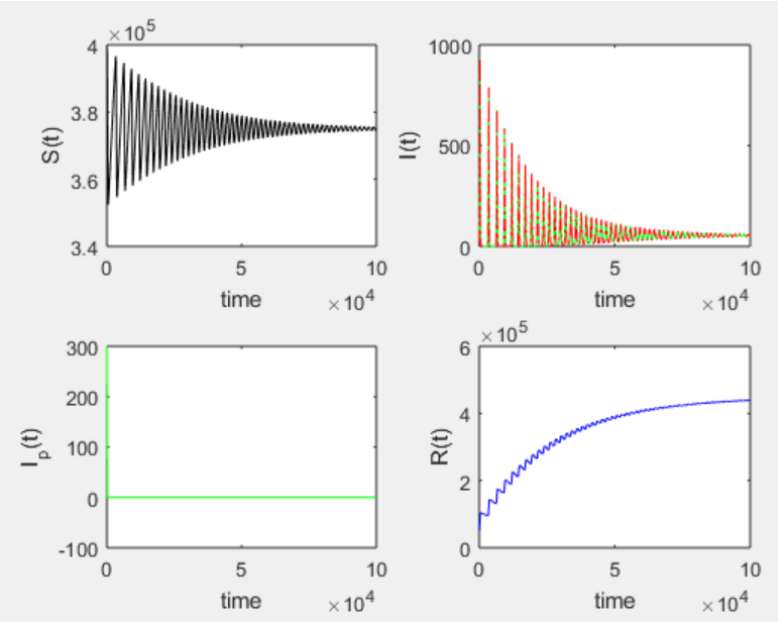

(a) Time series of $E_{1}$

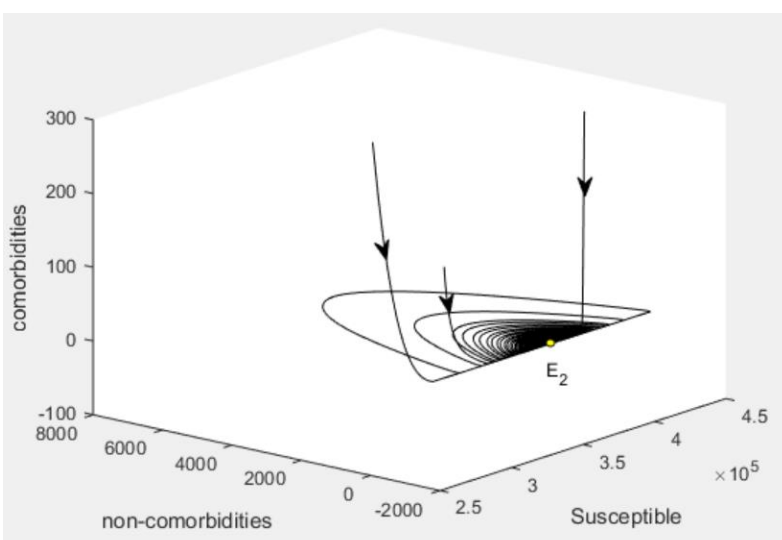

(b) Trajectories solution of $E_{1}$.

Figure 3 With the set of parameter values in (7), the non-comorbidities endemic equilibrium point $E_{1}$ is locally asymptotically stable.

\subsubsection{The comorbidities dominance equilibrium}

In this case, we set the parameter values as in (7) except $\alpha_{2}=0.1$. Thus, we have $R_{1}=2.19753<$ $R_{2}=3.2961$, so that $R_{0}=R_{2}=3.2961$ and the condition of Theorem 2.5(ii) is satisfied. So, in this case we have the unstable disease-free equilibrium point $E_{0}=(824,175,0,0,0)$, the unstable noncomorbidities endemic equilibrium $E_{1}=$ $(375,045.5,54.48,0,449,075.8)$, and the stable comorbidities endemic equilibrium $E_{2}=$ $(250,045.5,0,104.5,287,012.9)$. Figure 4 shows the time series and trajectories solution of the stable comorbidities endemic equilibrium point $E_{2}$.
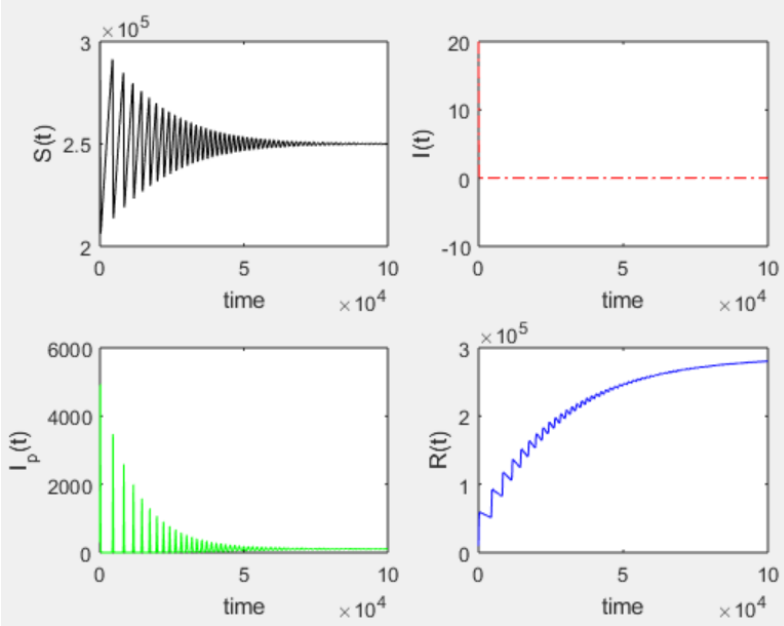

(a) Time series of $E_{2}$.

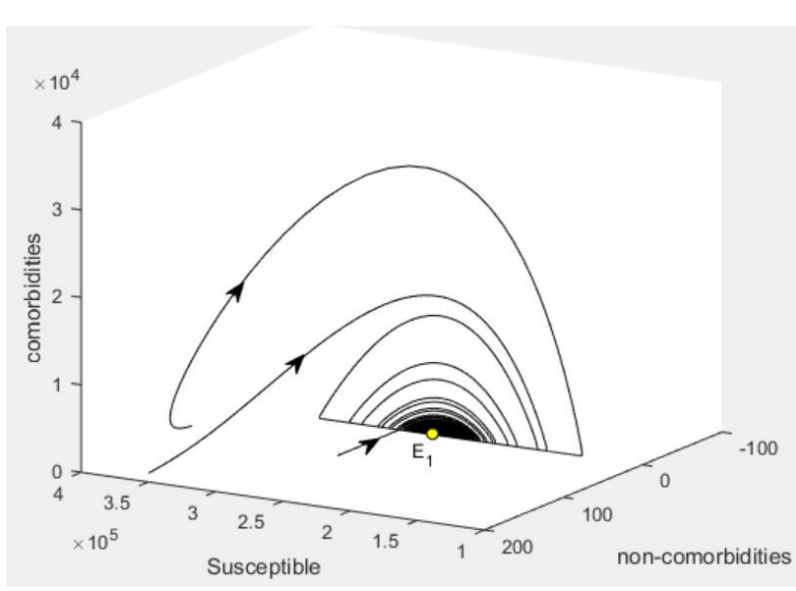

(b) Trajectories solution of $E_{2}$.

Figure 4 With the set of parameter values in (7) except $\alpha_{2}=0.1$, the non-comorbidities endemic equilibrium point $E_{2}$ is locally asymptotically stable.

\section{DISCUSSION AND FUTURE WORK}

In this paper, we discuss a susceptible-infectedrecovered (SIR) epidemic model involving two infective classes. The first class is the infected individuals who do not have comorbidities, while the second class is the individuals who have comorbidities. Comorbidities of the patients are factors that greatly affect the severity of the disease and often cause death. Numerical analysis and 
simulation have been carried out on the introduced model. Numerical simulation of this model is done by selecting suitable parameter values. The simulation also shows the effect of changing the parameter values on the dynamics of the disease transmission. We believe that this model can be implemented on real data, such as the data of COVID-19 transmission, so that strategies to overcome the disease can be determined and the disease can be eliminated immediately. Therefore, the model can still be developed and modified in the next research, so that it becomes more appropriate with the real condition of infectious diseases case, such as COVID-19.

\section{AUTHORS' CONTRIBUTIONS}

The authors confirm sole responsibility for the following: study conception, including literature study of biological fact of infectious diseases, mathematical theory such as non-linear differential equation system, dynamical system, and computation which give the analytical results supported by numerical results, manuscript writing and reviewing.

\section{ACKNOWLEDGMENTS}

The authors would like to thank the Ministry of Research, Technology and Higher Education of the Republic of Indonesia, Ahmad Dahlan University, and Sebelas Maret University who have supported this research.

\section{REFERENCES}

[1] https://covid19.kemkes.go.id/situasi-infeksiemerging/situasi-terkini-perkembangancoronavirus-disease-covid-19-20-juli-2021

[2] K. Prem, Y. Liu, T. W. Russell, et al. The effect of control strategies to reduce social mixing on outcomes of the COVID-19 epidemic in Wuhan, China: a modelling study, Lancet Public Health, 5(2020), E261-E270.

[3] Y. Wang, H. Kang, X. Liu, Z. Tong, Combination of RT-qPCR testing and clinical features for diagnosis of covid-19 facilitates management of Sars-Cov-2 outbreak, J. Med. Virol. 92(2020), 538-539.

[4] L. Lan, D. Xu, G. Ye, C. Xia, S. Wang, Y. Li, H. $\mathrm{Xu}$, Positive RT-PCR Test Results in Patients Recovered From COVID-19, JAMA, 323(2020), 1502-1503.

[5] L. Wang, J. Wang, H. Zhao, Y. Shi, K. Wang, P. $\mathrm{Wu}$, Modelling and assessing the effects of medical resources on transmission of novel coronavirus (COVID-19) in Wuhan, China, Math. Biosci. Eng. 17(2020), 2936.

[6] M.Z. Ndii \& Y.A. Adi, Modelling the transmission dynamics of covid-19 under limited resources. Communication in Mathematical Biology and Neurosciences, 2020, pp. 1-24.

[7] D. Aldila, Cost-effectiveness and backward bifurcation analysis on Covid-19 transmission model considering direct and indirect transmission, Commun. Math. Biol. Neurosci. 2020(2020), Article ID 49.

[8] D. Aldila, S.H.A. Khoshnaw, E. Safitri, et al. A mathematical study on the spread of COVID-19 considering social distancing and rapid assessment: The case of Jakarta, Indonesia, Chaos Solitons Fractals. 139(2020), 110042.

[9] C. Anastassopoulou, L. Russo, A. Tsakris, C. Siettos, Databased analysis, Modelling and forecasting of the Covid-19 outbreak, PLOS ONE, 15(2020), e0230405.COVID-19 UNDER LIMITED RESOURCES 23

[10] G. Giordano, F. Blanchini, R. Bruno, P.Colaneri, A. Di Filippo, A. Di Matteo, M. Colaneri, Modelling the COVID-19 epidemic and implementation of population-wide interventions in Italy, Nat. Med. 26(2020), 855-860.

[11] A. J. Kucharski, T. W. Russell, C. Diamond, et al. Early dynamics of transmission and control of Covid-19: a mathematical modelling study, Lancet Infect. Dis. 20(2020), 553-558.

[12] J. Hellewell, S. Abbott, A. Gimma, et al. Feasibility of controlling Covid-19 outbreaks by isolation of cases and contacts, Lancet Glob. Health, 8(2020), e488-e496.

[13] A. Sanyaolu, C. Okorie, A. Marinkovic et.al., Comorbidity and its Impact on Patients with COVID-19, SN Comprehensive Clinical Medicine, 2020, https://doi.org/10.1007/s42399020-00363-4

[14] O. Diekmann, H. Heesterbeek, T. Britton, Mathematical Tools for Understanding Infectious Disease Dynamics, Princeton University Press, 2013.

[15] J. Hale, Ordinary Differential Equations, Wiley, New York, 1969. 